\title{
Management and Pathophysiology of Functional Gastrointestinal Disorders
}

\author{
Shin Fukudo ${ }^{a}$ Hiroyuki Kuwano $^{\text {b }}$ Hiroto Miwac \\ a Department of Behavioral Medicine, Tohoku University Graduate School of Medicine, Miyagi, \\ ${ }^{b}$ Department of General Surgical Science, Gunma University Graduate School of Medicine, Maebashi, and \\ 'Division of Upper Gastroenterology, Department of Internal Medicine, Hyogo College of Medicine, Nishinomiya, Japan
}

\section{Key Words}

Functional dyspepsia $\cdot$ Functional gastrointestinal disorder $\cdot 5$-Hydroxytryptamine $\cdot$ Pathophysiology $\cdot$ Pharmacotherapy $\cdot$ Postprandial distress syndrome Transient receptor potential vanilloid-1 $\cdot$ Transient receptor potential A1 - Chronic intestinal pseudo-obstruction

\begin{abstract}
Since 2005, every annual meeting of the Japanese Gastroenterological Association has included a core symposium for functional gastrointestinal disorders. At the 6th annual meeting, the core symposium was 'Pathophysiology and New Treatment'. At the 7th annual meeting, the core symposium was 'Pathophysiology and Motility'. This review summarizes the papers presented at these meetings. At the 6th meeting, we recognized that Japanese researchers successfully produced and developed many agents that are safe and effective for the treatment of functional gastrointestinal disorders, such as 5-hydroxytryptamine receptor-associated compounds, lubiprostone, Japanese herbal medicine, and other drugs. Data were validated from a clinical as well as an experimental viewpoint. Findings included the effects of sumatriptan and nizatidine, acylated or des-acylated ghrelin, T-cell-activating anti-CD3 antibody, and transient receptor potential vanilloid-1. At the 7th meeting, not only functional dyspepsia and irritable bowel syndrome (IBS), but also nonerosive esophageal reflux disease (NERD) and chronic intestinal pseudo-obstruction were actively discussed from a motility viewpoint, including papers about sham feeding and
\end{abstract}

\section{KARGER}

Fax +41613061234 E-Mail karger@karger.ch www.karger.com
(C) 2012 S. Karger AG, Base 0012-2823/12/0852-0085\$38.00/0

Accessible online at: www.karger.com/dig gastric motility, genetic polymorphism and motility, the role of transient receptor potential A1 on gastric accommodation, esophageal motility and NERD, diagnosis and treatment of chronic intestinal pseudo-obstruction, immunological basis of motility in IBS, developing non-invasive colonic function test, and fecal distribution in IBS patients.

Copyright $\odot 2012$ S. Karger AG, Basel

\section{Introduction}

The field of the science of digestion is greatly expanding. Traditionally, digestive diseases were considered to be limited to the gastrointestinal (GI) tract, the hepatobiliary system, and the pancreas. Although the pathophysiological condition of digestive disease in the digestive organs is primarily important, significance of regulatory factors on and impact on the other systems from the digestive organs is gradually increasing in the society. Functional GI disorders (FGIDs) are the typical examples of how the digestive system plays a crucial role in the normal lives of humans regardless of their living area.

\section{Pathophysiology and New Treatment}

The 6th annual meeting of the Japanese Gastroenterological Association (February 2010 in Fukuoka, organized by Mitsuo Iida, Kyushu University) was chaired by Shin Fukudo (Tohoku University) and Hiroto Miwa (Hyogo 


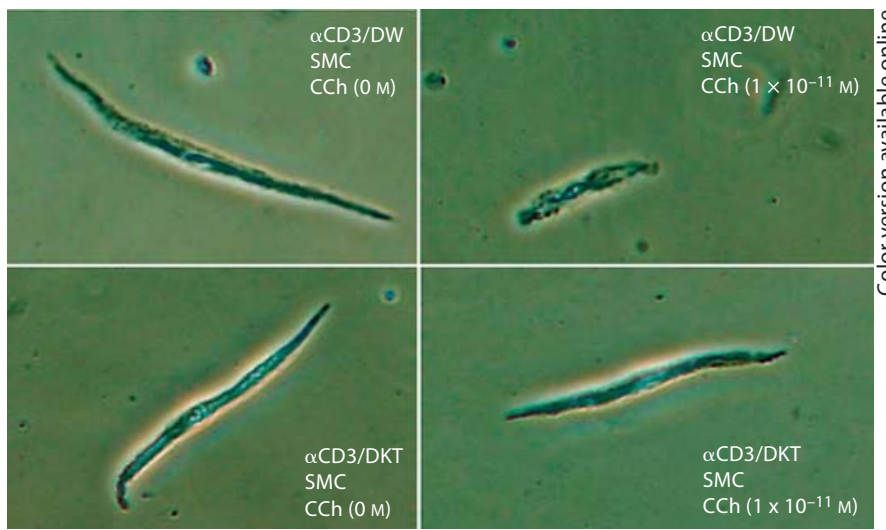

Fig. 1. Effect of DKT on $\alpha \mathrm{CD} 3$-induced hypercontractility of murine smooth muscle cells (SMC). Photomicrographs of SMC isolated from the small intestine of $\alpha \mathrm{CD} 3$-injected mice with or without DKT. The contraction was induced by addition of $10^{-11} \mathrm{M}$ carbamylcholine chloride (CCh) [from 9, with permission].

College of Medicine). The theme of the core symposium was 'Pathophysiology and New Treatment' and eight active researchers presented findings. Nakada et al. [1] reported that a significant difference in reduction of symptom scores between mosapride (5-hydroxytryptamine, $5-\mathrm{HT}_{4}$ receptor agonist) and teprenon was found in 618 dyspeptic patients in the Japan Mosapride Mega-Study. The responder rate to mosapride was superior to that of teprenon. Tomita et al. [2] described a cross-over trial of gastric emptying using ${ }^{99 \mathrm{~m}} \mathrm{Tc}-\mathrm{Sn}$-colloid scintigram that compared placebo, sumatriptan, and mosapride in 10 healthy subjects. Sumatriptan increased reservoir function of the stomach but delayed emptying. Mosapride increased both reservoir function and gastric emptying.

Futagami et al. [3] showed that administration of the histamine-2-receptor antagonist nizatidine significantly improved both gastric emptying and clinical symptoms in functional dyspepsia (FD) patients with impaired gastric emptying. Neither acylated nor des-acylated ghrelin levels in plasma were changed in FD patients treated with nizatidine compared with those treated with placebo. Another report [4] showed that acylated ghrelin levels were significantly lower in non-erosive esophageal reflux disease (NERD) and postprandial distress syndrome (PDS) patients than in healthy volunteers. Tominaga et al. [5] reported that disordered emptying was seen in 55.7\% (34/61) of FD patients. Among them, delay was seen in $67.6 \%$ $(23 / 34)$ and acceleration was detected in $32.4 \%$ (11/34). Impaired reservoir function was found in $49.2 \%$ (30/61) of patients, which was significantly associated with delayed and disordered (delay + acceleration) emptying. Symptoms in the motility disordered group tended to be more severe than in the normal group. They also use positron emission tomography with $\left[{ }^{11} \mathrm{C}\right] \mathrm{N}, \mathrm{N}$-dimethyl-2-(2-amino-4-cyanophenylthio)benzylamine [6]. In their report, FD patients showed higher binding potential of the 5-HT transporter in the thalamus and the midbrain than controls.

Mizukami et al. [7] tried to evaluate bowel motility and morphology in 146 patients with irritable bowel syndrome (IBS) by colonoscopy with the collapse-submergence (water navigation) method. In their observation, peristalsis persisted for $8 \mathrm{~min}$ or more in $67 \%$ of male patients with IBS with diarrhea (IBS-D) and 78\% of female patients with IBS-D; segmental type motility occurred in $48 \%$ of male patients with IBS with constipation (IBS-C) and 10\% of female patients with IBS-C after administration of an antispasmodic. Onodera et al. [8] examined the association of $\beta_{3}$-adrenoceptor and cholinergic receptor muscarinic-3 (CHRM3) polymorphisms with IBS. The $\beta_{3}$-adrenoceptor genotype frequencies of T/C in IBS patients were significantly higher compared with those in controls. The frequencies of the CHRM3 genotypes were significantly different between IBS patients with more than 3 years' disease duration and those with less than 3 years' disease duration.

Akiho and Nakamura [9] evaluated the effects of daikenchuto (DKT), a pharmaceutical-grade Japanese herbal medicine, on the hypercontractility of intestinal smooth muscle persisting after acute inflammation induced by a T-cell-activating anti-CD3 antibody $(\alpha C D 3)$. DKT ameliorated the $\alpha \mathrm{CD} 3$-induced muscle hypercontractility in both the muscle strips and smooth muscle cells (fig. 1). Although the influence of DKT on mRNA expressions was moderate, the protein expressions of interleukin (IL)-13 and IL-17 were significantly decreased. The observed modulation of cytokine expression and function by DKT may lead to the development of new pharmacotherapeutic strategies aimed at a wide variety of gut motor dysfunction disorders. Matsumoto et al. [10] found transient receptor potential vanilloid-1 (TRPV1) immunoreactivity in the mucosa, submucosal, and muscle layers and myenteric plexus in the rectum and distal colon. In contrast, TRPV1-positive axons were sparsely distributed in the transverse and proximal colon. Capsaicin induced a fast transient contraction, followed by a large long-lasting contraction in the rectum and distal colon. These authors also reported colocalization of immunoreactivity of TRPV1 and calcitonin gene-related peptide, substance $\mathrm{P}$, and neuronal nitric oxide synthase in the colorectum, suggesting regulation of colorectal function by TRPV1-expressing nerve cells (fig. 2) [11]. 
Fig. 2. Colocalization of TRPV1-positive nerve fibers with substance $P$ in mucosa (a-c), blood vessels (d-f), deep muscular plexus (g-i), circular muscle $(\mathbf{j}-\mathbf{I})$, myenteric plexus (m-o), and longitudinal muscle $(\mathbf{p}-\mathbf{r})$. Mouse rectum sections were double-labeled with TRPV1 (green) and substance P (red). Labeling for TRPV1 and substance $P$ is shown separately $(\mathbf{a}, \mathbf{d}, \mathbf{g}, \mathbf{j}$, $\mathbf{m}, \mathbf{p})$ and $(\mathbf{b}, \mathbf{e}, \mathbf{h}, \mathbf{k}, \mathbf{n}, \mathbf{q})$, respectively, and merged (c, f, i, l, o, $\mathbf{r})$. Arrows indicate the colocalization of TRPV1 immunoreactivity with substance $\mathrm{P}$ immunoreactivity. Arrowheads indicate substance P-positive nerve fibers not colocalized with TRPV1. Scale bars are $20 \mu \mathrm{m}$ [from 10, with permission].

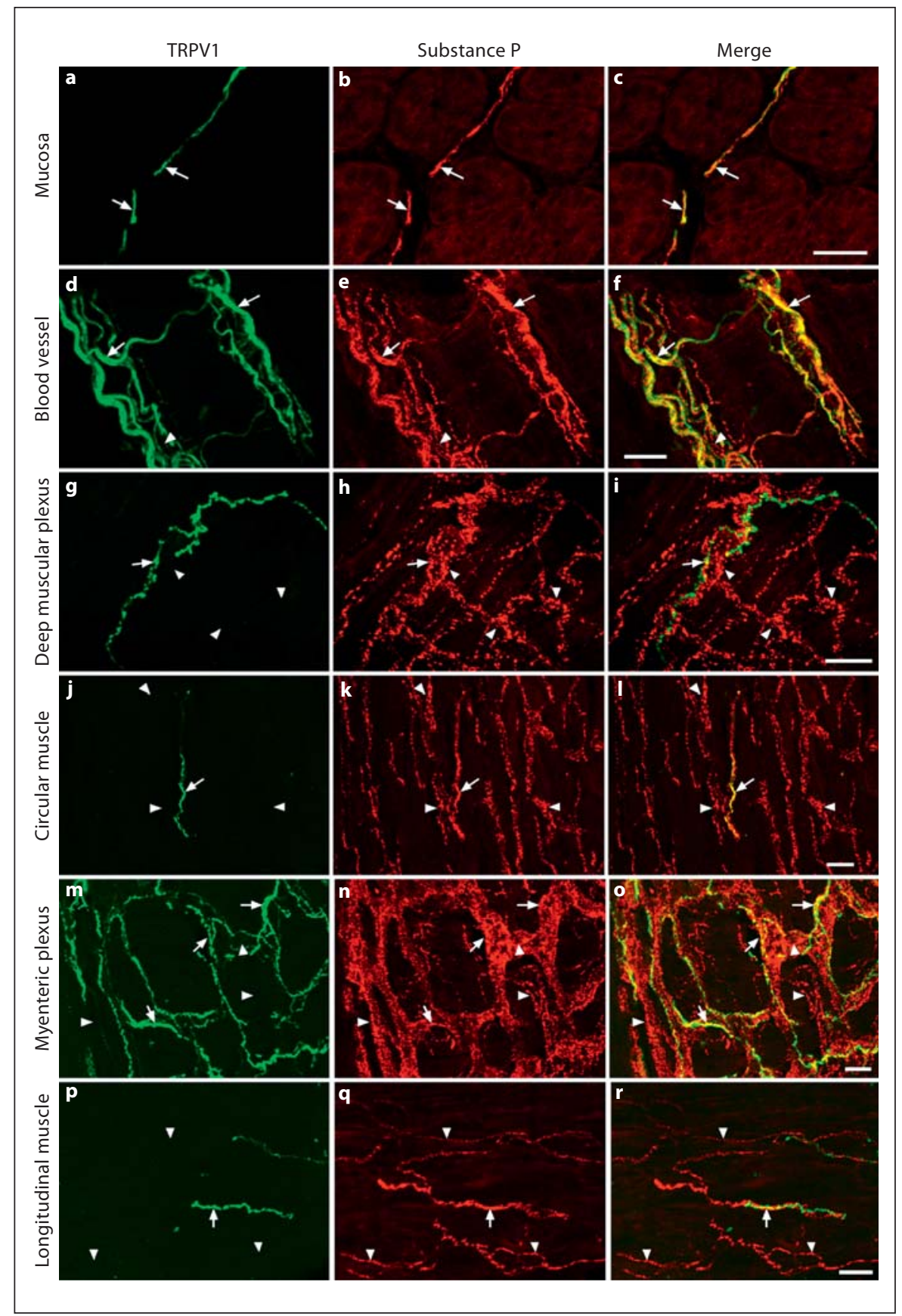

\section{Pathophysiology and Gastrointestinal Motility}

At the 7th annual meeting (February 2011 in Kyoto, organized by Toshikazu Yoshikawa, Kyoto Prefectural University of Medicine), the title of the core symposium was 'Functional Gastrointestinal Disorders (FGID); Pathophysiology and Motility'. This meeting was chaired by Hiroyuki Kuwano (Gumma University) and Hiroto
Miwa. At this meeting, ten researchers presented their papers at the symposium. Ohno et al. outlined basic GI motility and ways in which it can be evaluated. Choosing an appropriate evaluation method and proper interpretation are essential. Manabe et al. [12] reported results of a prospective study of the gastric response to modified sham feeding in patients with PDS. First, cardiovascular autonomic function was assessed by spectral analysis of 
RR interval variability. Antral contraction was then evaluated by ultrasonography after modified sham feeding was performed to stimulate the cephalic phase of vagal activity. Autonomic abnormalities affecting the cephalic phase of vagal activity may be important in the pathogenesis of FD. Shibata et al. presented the correlation between 5-HT, brain-derived neurotrophic factor, ghrelin, neuropeptide $\mathrm{Y}$, and T1R3 gene polymorphism and dyspeptic symptoms in a Japanese population. They suggested that the $5-\mathrm{HT}_{1 \mathrm{~A}}$ receptor polymorphism is associated with the appearance of FD. The role of genetics in the development of dyspepsia needs further evaluation.

Kondo et al. [13] presented the role of transient receptor potential A1 (TRPA1) in gastric adaptive relaxation. They developed a new gastric pressure measurement model that can be performed while subjects are awake using a guinea pig, and enabled the measurement of gastric adaptive relaxation under physiological conditions. TRPA1 raised the intragastric pressure via the cholinergic pathway and negatively controlled adaptive relaxation. Iwakiri et al. [14] presented the characteristics of symptomatic reflux episodes in patients with NERD who have a positive symptom index while receiving proton pump inhibitor therapy. Proximal reflux is more likely to be associated with reflux symptoms, irrespective of the acidity of the refluxate or the duration of proximal reflux episodes. In NERD patients who have a positive symptom index on double-dose proton pump inhibitor therapy, the high proximal extent of refluxate is a major factor associated with reflux perception. The lack of secondary peristalsis may be related to the rise of proximal reflux. Funaki et al. reported that functional heartburn was defined in Rome III in 2006; however, there were difficulties in diagnosing all functional heartburn based on this definition. They evaluated esophageal function and pointed out that many esophageal physiologic dysfunctions were included in the diagnosis of functional heartburn based on the definition of Rome III, and determined that it is necessary to define functional heartburn in a more narrow sense to make an appropriate diagnosis.

Sakamoto et al. reported on the current state of diagnosis and treatment of chronic intestinal pseudo-obstruction, based on the result of the survey of the research group of the Ministry of Health, Labor and Welfare. They indicated that further research and surveys conducted by medical institutions nationwide, including surgery facilities, would be needed to determine the etiology of chronic intestinal pseudo-obstruction and rapid establishment of diagnostic standards and treatments. Akiho et al. [15] showed that understanding the underlying immunological basis of the altered GI motor dysfunction in IBS by considering the role of the Th1/Th2 balance or Th17 cytokines may ultimately lead to new therapeutic strategies for IBS.

Okahisa et al. established a simple and non-invasive evaluation method of assessing colonic function by measuring electric potential. They reported that this method is useful for the evaluation of various medicines under development, as well as the diagnosis, detailed classification, and treatment of the FGID. Kusunoki et al. [16] examined fecal distribution in the colon in uninfected IBS and post-infectious IBS patients using ultrasonography. They showed that early onset post-infectious IBS and mental instability such as depression and anxiety were major risk factors. They also suggested the possibility that the strength of the inflammation of long-term IBS could be a risk factor as well.

\section{Perspective of FGID Research}

In these sessions, pathophysiology of FGID was discussed by front-line Japanese researchers in this field. Considering that the mainstream of GI research has been focused on morphological studies, presentation of such high-quality and unique papers encourages us to further perform more research in this field. Increased understanding of pathophysiology of FGID will facilitate drug discovery. It was noted that Japanese researchers successfully produced and developed many agents that are safe and effective for FGID. They are ramosetron for IBS-D [17], lubiprostone for chronic idiopathic constipation, and IBS-C [18], tandospirone for FD [19], and acotiamide for FD [20]. Besides, several Japanese agents for FGIDs are available in Asia. Mosapride is available as a $5-\mathrm{HT}_{4}$ receptor agonist for clinical use. This agent is predominantly prescribed for patients with FD, but a recent study clarified its stimulatory effect on the colorectum in patients with IBS [21]. Further data on clinical trials of new agents for FGID from Japan and the other Asian countries are warranted.

\section{Direction of Research in the Future}

Like researches listed in this article, basic and clinical investigations are indispensable for care and cure for FGIDs. Regarding the era of genome-wide association studies, nature or nurture problems of FGIDs will be solved scientifically in the future. Common genes and common risk stimuli for FGIDs regardless of the ethnic factors and/or cultural issues may be present. In addition, 
some different features of FGIDs, some differences in genes, and differences in cultural behaviors including the dietary factors/gut microbiota in the different countries will provide us important keys to open the black box of FGIDs. The theme of the next core symposium at the 8th JGA meeting in 2012 will be 'Functional Gastrointestinal Disorders (FGID): Pathophysiology and Sensation'.

\section{Conclusion}

Molecular mechanism and whole-body analyses for FGIDs are actively and continuously studied in Japan. Further research and development of treatment for FGID are warranted.

\section{Acknowledgements}

This research was supported by a Grant-in-Aid for Scientific Research from the Ministry of Education, Science, and Culture of Japan, and a Grant-in-Aid for Scientific Research from the Ministry of Health, Welfare, and Labor of Japan.

\section{Disclosure Statement}

The authors declare that there is no conflict of interest in relation to the contents of this article.

\section{References}

- 1 Nakada K, Hongo M, Harasawa S, Mine T, Sasaki I, Matsueda K, Kusano M, Hanyuu N, Shibata C: How the subgroups in functional dyspepsia affect the quality of life and therapeutic approaches. Experience from Japanese Mosapride Mega-Study (JMMS). Gastroenterology 2009;136(suppl 1):A183.

2 Tomita T, Okugawa T, Toyoshima F, Yamasaki T, Sakurai J, Tanaka J, Morita T, Kim Y, Oshima T, Hori K, Watari J, Matsumoto T, Kashiwagi T, Miwa $\mathrm{H}: 5-\mathrm{HT}_{4}$ receptor agonist, a mosapride citrate, facilitates both gastric accommodation and emptying in healthy volunteers. The 17th United European Gastroenterological Week, London, UK, 2009, 11.25 .

-3 Futagami S, Shimpuku M, Kawagoe T, Kusunoki M, Ueki N, Miyake K, Iwakiri K, Sakamoto C: Nizatidine administration improves clinical symptoms and gastric emptying of the patients with functional dyspepsia accompanying with impaired gastric emptying. Gastroenterology 2011;140(suppl 1):S230.

-4 Shindo T, Futagami S, Hiratsuka T, Horie A, Hamamoto T, Ueki N, Kusunoki M, Miyake K, Gudis K, Tsukui T, Iwakiri K, Sakamoto C: Comparison of gastric emptying and plasma ghrelin levels in patients with functional dyspepsia and non-erosive reflux disease. Digestion 2009;79:65-72.

5 Tominaga K, Higuchi K, Ochi M, Kadouchi K, Kawamura E, Tanigawa T, Shiba M, Watanabe T, Fujiwara Y, Oshitani N, Shiomi S, Arakawa T: Concurrent assessment of reservoir and emptying of the stomach for dyspepsia patients. Hepatogastroenterology 2008;55:744-749.

6 Tominaga K, Tsumoto C, Ataka S, Machida $\mathrm{H}$, Okazaki H, Yamagami H, Tanigawa T, Watanabe K, Watanabe T, Fujiwara Y, Shiomi S, Watanabe Y, Arakawa T: Overlapped heartburn/regurgitation amplifies abdominal pain and indigestion via common central serotonin neurotransmission disorders in patients with functional dyspepsia. Gastroenterology 2010;138(suppl 1):S370.

7 Mizukami T, Suzuki H, Hibi T: Classification and treatment of irritable bowel syndrome based on colonoscopy abnormal bowel motility type and abnormal bowel morphology type. Gastroenterology 2010;138 (suppl 1):S233.

8 Onodera S, Chiba T, Sugai T, Habano W: A genetic association between $\beta_{3}$-aderenoceptor and cholinergic receptor muscarinic- 3 polymorphisms in irritable bowel syndrome. Hepatogastroenterology 2011;58:110-111.

-9 Akiho H, Nakamura K: Daikenchuto ameliorates muscle hypercontractility in a murine T-cell-mediated persistent gut motor dysfunction model. Digestion 2011;83:173-179.

10 Matsumoto K, Kurosawa E, Terui H, Hosoya T, Tashima K, Murayama T, Priestley JV, Horie S: Localization of TRPV1 and contractile effect of capsaicin in mouse large intestine: high abundance and sensitivity in rectum and distal colon. Am J Physiol Gastrointest

Liver Physiol 2009;297:G348-G360.
Matsumoto K, Hosoya T, Tashima K, Namiki T, Murayama T, Horie S: Distribution of transient receptor potential vanilloid-1 channel-expressing nerve fibers in mouse rectal and colonic enteric nervous system: relationship to peptidergic and nitrergic neurons. Neuroscience 2011;172:518-534.

12 Manabe N, Nakamura K, Hara M, et al: Impaired gastric response to modified sham feeding in patients with postprandial distress syndrome. Neurogastroenterol Motil 2011;23:215-219, e112.

13 Kondo T, Oshima T, Obata K, et al: Role of transient receptor potential Al in gastric nociception. Digestion 2010;82:150-155.

14 Iwakiri K, Sano H, Tanaka Y, et al: Characteristics of symptomatic reflux episodes in pa- tients with non-erosive reflux disease who have a positive symptom index on proton pump inhibitor therapy. Digestion 2010;82:156-161.

15 Akiho H, Ihara E, Nakamura K: Low-grade inflammation plays a pivotal role in gastrointestinal dysfunction in irritable bowel syndrome. World J Gastrointest Pathophysiol 2010;1:97-105.

16 Kusunoki H, Kamada T, Sato M, et al: Ultrasonographic assessment of sigmoid colon in patients with irritable bowel syndrome (in Japanese). Nihon Rinsho 2006;64:1461-

1466.
17 Miyata K, Ito H, Fukudo S: Involvement of the 5- $\mathrm{HT}_{3}$ receptor in $\mathrm{CRH}$-induced defecation in rats. Am J Physiol 1998;274:G827-G831.

18 Fukudo S, Hongo M, Kaneko H, Ueno R: Efficacy and safety of oral lubiprostone in constipated patients with or without irritable bowel syndrome: a randomized, placebocontrolled and dose-finding study. Neurogastroenterol Motil 2011;23:544-e205.

19 Miwa H, Nagahara A, Tominaga K, Yokoyama T, Sawada Y, Inoue K, Ashida K, Fukuchi T, Hojo M, Yamashita H, Tomita T, Hori K, Oshima T: Efficacy of the $5-\mathrm{HT}_{1 \mathrm{~A}}$ agonist tandospirone citrate in improving symptoms of patients with functional dyspepsia: a randomized controlled trial. Am J Gastroenterol 2009; 104:2779-2787.

20 Matsueda K, Hongo M, Tack J, Aoki H, Saito Y, Kato H: Clinical trial: dose-dependent therapeutic efficacy of acotiamide hydrochloride (Z-338) in patients with functional dyspepsia - $100 \mathrm{mg}$ tid is an optimal dosage. Neurogastroenterol Motil 2010;22:618-e173.

-21 Kanazawa M, Watanabe S, Tana C, Komuro H, Aoki M, Fukudo S: Effect of 5- $\mathrm{HT}_{4}$ receptor agonist mosapride citrate on rectosigmoid sensorimotor function in patients with irritable bowel syndrome. Neurogastroenterol Motil 2011;23:754-e332. 